\title{
Beamforming with Limited Feedback in Amplify-and-Forward Cooperative Networks
}

\author{
Yi Zhao, Raviraj Adve and Teng Joon Lim \\ Dept. of Electrical and Computer Engineering, \\ University of Toronto, \\ M5S3G4, Canada. \\ E-mail: zhaoyi, rsadve, limtj@comm.utoronto.ca.
}

\begin{abstract}
A relay selection approach has previously been shown to outperform repetition-based scheduling for both amplify-and-forward (AF) and decode-and-forward (DF) cooperative networks. The selection method generally requires some feedback from the destination to the relays and the source, raising the issue of the interplay between performance and feedback rate. In this paper, we treat selection as an instance of limitedfeedback distributed beamforming in cooperative AF networks, and highlight the differences between transmit beamforming in a traditional multi-input single-output (MISO) system and the distributed case. Specifically, Grassmanian line packing (GLP) is no longer the optimal codebook design, and orthogonal codebooks are no longer equivalent to each other. We derive the high signal-to-noise ratio expressions for outage probability and probability of symbol error for unlimited-feedback and selection schemes. The gap in performance between unlimited-feedback and selection beamforming is found analytically to grow rapidly with the number of relays. We compare the selection protocol to a limited-feedback distributed beamformer that assigns codebooks based on the Generalized Lloyd Algorithm (GLA), and one that uses random beam-vectors. The main conclusion is that the performance improvement to be seen using the very complex GLA is small, and that many more feedback bits are required with random beamforming than selection for the same performance. These results indicate that the selection protocol is a very attractive protocol with low-complexity that provides excellent performance relative to other known methods.

Index terms: beamforming, limited feedback, selection, amplify-and-forward, wireless cooperative networks.
\end{abstract}

\section{INTRODUCTION}

Cooperative diversity [1] is an important concept for achieving spatial diversity in distributed wireless networks where antenna arrays are not available at each node. Amplify-andForward (AF) relaying, where a relay node simply amplifies its received signal from the source node and retransmits, is perhaps the most studied cooperative diversity scheme due to its low complexity. Given multiple relay nodes in the system, retransmissions can take place in orthogonal channels (e.g., time slots) in a repetition-based (RB) protocol [1].

The bandwidth efficiency of RB scheduling is limited as it only uses $1 /(m+1)$ of the available channel bandwidth for information transmission, where $m$ is the number of relays. A selection scheme was introduced in [2]-[4] to make more efficient use of channel resources. It was proven in these works that selection outperforms RB scheduling in AF networks in terms of achieving higher average throughput, lower outage probability and lower error probability, while maintaining the same diversity order, at the cost of only $\log _{2} m$ bits of feedback. In fact, the selection scheme is a special case of beamforming with $\log _{2} m$ bits of feedback and the columns of the $m \times m$ identity matrix used as the beamformer codebook. One should note that in [5] the authors present an alternative selection approach, but one that may result in packet collisions at the receiver.

Optimal relay beamforming with unlimited feedback (BUF) has been studied in [6]. Although the B-UF scheme seems impractical with its requirement for perfect phase synchronization among relays, it provides a performance upper bound for practical beamforming schemes with limited feedback. In this paper, we derive the outage probability and probability of error for B-UF, and compare those expressions with the ones corresponding to the selection scheme [3], [4] to find the performance gap between B-UF and selection.

With limited feedback, distributed beamforming over multiple relays appears equivalent to MISO beamforming. In the latter context, Grassmanian Line Packing (GLP) provides the optimal codebook design for both average received signal to noise ratio (SNR) [7] and outage probability [8]. However, because AF relays include some noise amplification, a different solution is required. A numerical solution is developed here to find the optimal beamforming codebook. Aiming for complexity reduction, codebooks of random beamforming vectors were introduced in [9], [10] as a practical implementation of limited feedback beamforming. In this paper, we show that, to achieve the same performance, B-RC (beamforming with random codebooks) needs significantly more feedback than the selection scheme. In addition, all beamforming schemes require synchronization among all relay nodes. Therefore, selection relaying may be the most attractive currently known protocol for AF networks with limited feedback.

The rest of the paper is organized as follows. We introduce the system model in Section II. In Section III, we obtain the outage and error probabilities for the B-UF and selection schemes. In Section IV, we compare the performance of selection scheme to B-OC and B-RC. Finally, we draw some conclusions in Section V.

\section{SySTEM MODEL}

Consider a wireless system where a source node transmits to a destination with the help of $m$ relay nodes. As in [1], 
each transmission block is divided into two non-overlapping phases in time. In Phase I the source node transmits the unit power signal $x$ to the destination and relay nodes. The received signals at relay node $i$ and the destination are

$$
\begin{aligned}
r_{i} & =\sqrt{E_{s}} h_{s, i} x+n_{s, i}, \\
r_{d, 1} & =\sqrt{E_{s}} h_{s, d} x+n_{s, d},
\end{aligned}
$$

where $E_{s}$ is the transmitted symbol energy used at the source node. $h_{s, i}$ and $h_{s, d}$ are complex Gaussian coefficients of the source-relay and source-destination channels, respectively. $n_{s, i}$ and $n_{s, d}$ are the additive white Gaussian noise (AWGN) in the corresponding channels. We assume that all the noise contributions are independent and identically distributed (i.i.d.) with single-sided power spectral density $N_{0}$.

In Phase II, AF relays normalize and retransmit their received signals. The relay nodes jointly beamform to forward data to the destination; selection, wherein only one "best" relay node participates, is a special case of beamforming with the beamforming vector chosen from columns of the identity matrix. The signal to be transmitted from relay $i$ is thus

$$
x_{i}=\frac{r_{i}}{\sqrt{\mathcal{E}\left\{\left|r_{i}\right|^{2}\right\}}}=\frac{\sqrt{E_{s}} h_{s, i} x+n_{s, i}}{\sqrt{E_{s}\left|h_{s, i}\right|^{2}+N_{0}}}
$$

All $m$ nodes transmit simultaneously to the destination, with the $i$-th relay weighting $x_{i}$ with $w_{i}$, a complex beamforming weight. Thus, the received signal at the destination is

$$
\begin{aligned}
& r_{d, 2}=\sum_{i=1}^{m} \sqrt{E_{r}} w_{i} h_{i, d} x_{i}+n_{d} \\
= & \sum_{i=1}^{m} \frac{\sqrt{E_{s} E_{r}} w_{i} h_{s, i} h_{i, d} x}{\sqrt{E_{s}\left|h_{s, i}\right|^{2}+N_{0}}}+\sum_{i=1}^{m} \frac{\sqrt{E_{r}} w_{i} h_{i, d} n_{s, i}}{\sqrt{E_{s}\left|h_{s, i}\right|^{2}+N_{0}}}+n_{d} \\
= & \sum_{i=1}^{m} w_{i} \tilde{h}_{i} x+\tilde{n}_{d},
\end{aligned}
$$

where $E_{r}$ is the total transmitted symbol energy shared among relay nodes, $h_{i, d}$ is the complex Gaussian channel between relay $i$ and the destination, and $n_{d}$ is the AWGN at the destination node in Phase II. To keep the total energy used by all relays at $E_{r}$, the beamformer weights must satisfy $\sum_{i=1}^{m}\left|w_{i}\right|^{2}=1$. Note that beamforming schemes (other than selection) require phase synchronization across relays, an issue of practical importance.

In (4) we defined an equivalent channel and noise term to simply the expression. The equivalent channel through relay $i$ is

$$
\tilde{h}_{i}=\frac{\sqrt{E_{s} E_{r}} h_{s, i} h_{i, d}}{\sqrt{E_{s}\left|h_{s, i}\right|^{2}+N_{0}}},
$$

and equivalent noise $\tilde{n}_{d}$ is circularly symmetric Gaussian distributed as:

$$
\tilde{n}_{d} \sim \mathcal{C N}\left(0, \quad\left(1+\sum_{i=1}^{m}\left|w_{i}\right|^{2}\left|H_{i i}\right|^{2}\right) N_{0}\right),
$$

where

$$
H_{i i}=\frac{\sqrt{E_{r}} h_{i, d}}{\sqrt{E_{s}\left|h_{s, i}\right|^{2}+N_{0}}} .
$$

Maximal Ratio Combining (MRC) of the received signals over the two phases provides sufficient statistics for detection. The SNR in the decision statistic can be shown to be

$$
\gamma_{r}=E_{s}\left|h_{s, d}\right|^{2} \gamma_{t}+\frac{\left|\sum_{i=1}^{m} w_{i} \tilde{h}_{i}\right|^{2} \gamma_{t}}{1+\sum_{i=1}^{m}\left|w_{i} H_{i i}\right|^{2}},
$$

where $\gamma_{t}=1 / N_{0}$ is the equivalent system SNR since it is proportional to all the transmit and receive SNRs at all the nodes in the system.

\section{Optimal Beamforming And Selection Schemes}

\section{A. Optimal AF Beamforming with Unlimited Feedback}

In [6], the ideal scenario of zero channel estimation error and infinite feedback channel bandwidth is assumed, and hence $w_{i}$ 's can be calculated using exact instantaneous channel state information (CSI) at the destination and fed back to the relays without error. This yields the (unfortunately impractical) B-UF scheme and serves as a loose upper bound on performance for all beamforming schemes with limited feedback. In this section, we derive the outage and error probabilities for BUF, at high SNR.

The first term in the total SNR expression of (8) depends on source-destination channel alone. Hence only the SNR in Phase II (the second term in (8)) impacts the beamformer design. Stacking $w_{i}$ 's and $\tilde{h}_{i}$ 's into column vectors $\mathbf{w}$ and $\mathbf{h}$, respectively, and defining a diagonal matrix $\mathbf{H}$ whose $i$ th diagonal element is $H_{i i}$, we can rewrite the second term in (8) as

$$
\gamma_{r, 2}=\frac{\mathbf{w}^{\dagger} \mathbf{h} \mathbf{h}^{\dagger} \mathbf{w}}{\mathbf{w}^{\dagger}\left(\mathbf{I}+\mathbf{H H}^{\dagger}\right) \mathbf{w}} \gamma_{t},
$$

where $(\cdot)^{\dagger}$ denotes the Hermitian or conjugate transpose.

Maximizing $\gamma_{r, 2}$ over $\mathbf{w}$ (see [11]), with the energy constraint $\|\mathbf{w}\|_{2}=1$, yields the optimal beamforming vector with unlimited feedback as

$$
\mathbf{w}^{*}=\frac{\left(\mathbf{I}+\mathbf{H H}^{\dagger}\right)^{-1} \mathbf{h}}{\left\|\left(\mathbf{I}+\mathbf{H} \mathbf{H}^{\dagger}\right)^{-1} \mathbf{h}\right\|_{2}},
$$

where $\|\cdot\|_{2}$ denotes the 2-norm.

Note that the beamforming vector $\mathbf{w}$ not only determines the received signal power, but also contributes to noise amplification, as shown in (8). As a result, (10) verifies that the optimal beamforming vector differs significantly from the matched filtering solution in traditional MISO systems. We can therefore expect that the beamforming codebook design with limited feedback is quite different from the Grassmanian approach for MISO systems [7].

Substituting (10) and (9) into (8), we obtain the received SNR of the optimal beamforming AF network as

$$
\gamma_{r}^{o p t}=E_{s}\left|h_{s, d}\right|^{2} \gamma_{t}+\sum_{i=1}^{m} \frac{E_{s} E_{r}\left|h_{s, i}\right|^{2}\left|h_{i, d}\right|^{2}}{E_{s}\left|h_{s, i}\right|^{2}+E_{r}\left|h_{i, d}\right|^{2}+N_{0}} \gamma_{t}
$$

Note that, interestingly, this received SNR expression has the same form as in TDMA scheduled AF systems [4, Eq.(1)]. Therefore, the results in [3], [4] directly lead to: 
Lemma 1: At high SNRs the outage probability and probability of error of the optimal beamforming with unlimited feedback AF network are given by

$$
\begin{aligned}
P_{\text {out }}^{\text {opt }}\left(\gamma_{t}\right) & =\frac{\zeta}{m !(m+1)}\left(\frac{2^{(m+1) R}-1}{\gamma_{t}}\right)^{m+1}, \\
P_{e}^{\text {opt }}\left(\gamma_{t}\right) & =\frac{\zeta(2 m+1) !}{m !(m+1) !\left(2 c \gamma_{t}\right)^{m+1}},
\end{aligned}
$$

where

$$
\zeta=\frac{1}{E_{s} \sigma_{s, d}^{2}} \prod_{i=1}^{m}\left(\frac{1}{E_{s} \sigma_{s, i}^{2}}+\frac{1}{E_{r} \sigma_{i, d}^{2}}\right)
$$

is a constant determined by transmission power and channel variances $\sigma_{s, d}^{2}, \sigma_{s, i}^{2}$ and $\sigma_{i, d}^{2}, R$ is the target transmission rate and $c$ is a constant determined by the modulation scheme.

Proof: Refer to [3], [4].

\section{B. Selection AF Networks}

The idea of an AF network with relay selection was introduced in [3] as an improvement to the conventional TDMA-based AF networks. The high-SNR approximation of the outage probability and probability of error of the selection AF network are [3], [4]

$$
\begin{aligned}
P_{\text {out }}^{s}\left(\gamma_{t}\right) & =\frac{\zeta}{(m+1)}\left(\frac{2^{(m+1) R}-1}{\gamma_{t}}\right)^{m+1}, \\
P_{e}^{s}\left(\gamma_{t}\right) & =\frac{\zeta(2 m+1) !}{(m+1) !\left(2 c \gamma_{t}\right)^{m+1}},
\end{aligned}
$$

leading to

Theorem 1: The SNR gap ${ }^{1}$ between the selection AF scheme and the B-UF scheme is $\frac{10}{m+1} \log _{10} m ! \mathrm{dB}$.

Proof: From (12), (13) and (15), (16) with the same transmit SNR, the performances of the two schemes satisfy

$$
\frac{P_{o u t}^{s}}{P_{o u t}^{o p t}}=\frac{P_{e}^{s}}{P_{e}^{o p t}}=m !
$$

Since the high SNR approximation of the two schemes are parallel lines with slope $-(m+1) / 10$ in Log-dB scale, the SNR difference for the two schemes to achieve the same performance is therefore

$$
\delta_{\gamma}=\frac{\log m !}{(m+1) / 10}=\frac{10}{m+1} \log _{10} m ! \quad(\mathrm{dB}) .
$$

Corollary 1: As the number of relay nodes $m$ increases, the asymptotic SNR gap between selection AF and B-UF is $10 \log _{10} \mathrm{~m} / \mathrm{e} \mathrm{dB}$.

Proof: The result can be derived by applying Stirling's formula $m ! \approx \sqrt{2 \pi m} e^{-m} m^{m}$ to the results of Theorem 1 and retaining only the most significant term.

The gap shown in Theorem 1 is a quickly increasing function of $m$ and may seem to be severe in large networks. For instance, some examples are given in Table I. However, the optimal beamforming scheme is highly impractical for

\footnotetext{
${ }^{1}$ Defined analogously to SNR gap to capacity. In this case, it refers to the difference in SNR required to achieve the same performance with selection $\mathrm{AF}$ and with $\mathrm{B}-\mathrm{UF}$.
}

TABLE I

Performance Loss by Selection over Optimal Beamforming

\begin{tabular}{|c|c|c|c|c|c|}
\hline m & 2 & 3 & 6 & 10 & 20 \\
\hline Loss (in dB) & 1 & 1.95 & 4.08 & 5.96 & 8.76 \\
\hline
\end{tabular}

any realistic application, especially when $m$ is large, since it involves feed back of $m$ complex numbers in real time and strict synchronization. Therefore we treat it as a performance bound only and compare more practical schemes using their performance loss to this optimal one.

\section{BEAMForming With Limited FEedBACK}

In Section III we treated the selection AF scheme as one special case of beamforming with limited feedback and studied its performance loss in relation to the B-UF scheme. Clearly, this loss can be reduced by increasing the amount of feedback. In this section, we study the performance of AF beamforming schemes as a function of feedback available. In such a network, a codebook whose size is determined by the amount of feedback is first established at both the destination and the relay nodes. For each transmission, the destination selects the optimal codeword based on the CSI, and feeds back its index to the relays. The relays perform beamforming using the corresponding codeword as the weight vector.

\section{A. Optimal Codebook Design}

Optimal transmit beamforming codebook design has been studied in the context of single-user MISO (multi-input singleoutput) systems [7], [8], [12], where GLP provided the optimal codebook design for both average received SNR [7] and outage probability [8]. In the AF networks, however, GLP is no longer optimal due to the noise amplification by relay nodes. The Generalized Lloyd Algorithm (GLA) [13] can still be used in the optimal codebook design. In particular, assuming $B$ bits of feedback, the algorithm starts with a set of randomly selected $2^{B}$ vectors, and repeats the following two steps until convergence:

1) For each codeword in the current codebook, find the region in $\mathbb{C}^{m}$ for which it is the $\gamma_{r}$-maximizing beamforming vector.

2) For each region, find a new codeword to replace the current one by maximizing the average $\gamma_{r}$ over that region.

Due to the complex form of $\gamma_{r}$, only numerical results are available for AF relays (shown later in simulations), even for the special case of i.i.d channels among the network. We are particularly interested in $\log _{2} m$ bits of feedback, for the selection scheme is a possible candidate in this case. Unlike in MISO systems, where any orthogonal basis of $m$-dimensional space is an optimal codebook, selection (identity codebook) is the unique optimal for AF beamforming, as shown in Figure 1. From the figure we can see that the selection scheme significantly outperforms the randomly chosen orthogonal codebook, although the feedback rates are the same. The reason for the uniqueness is straightforward: although all orthogonal 


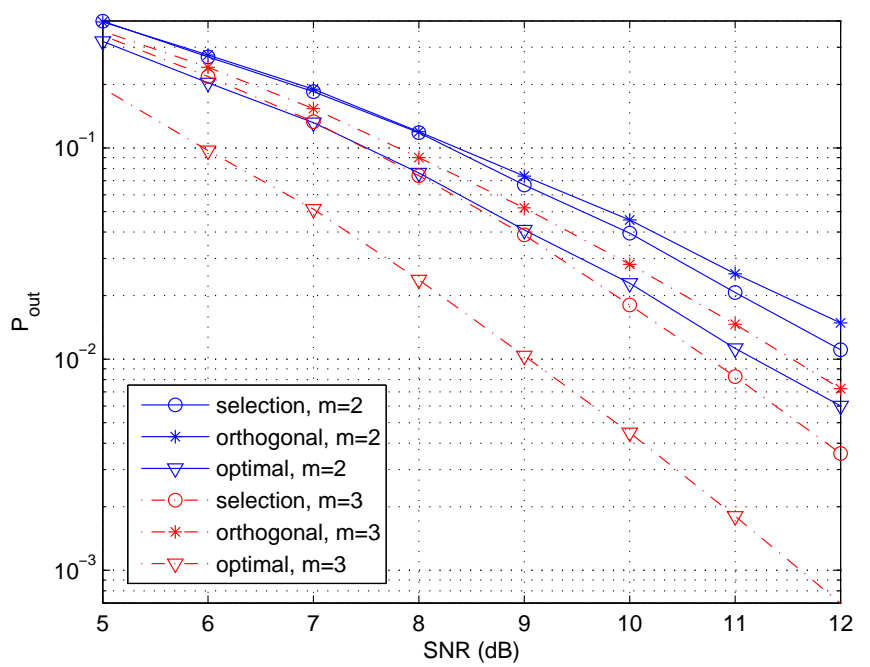

Fig. 1. Outage Probabilities for Selection, Random Orthogonal Codebook and Optimal Beamforming, $R=1$

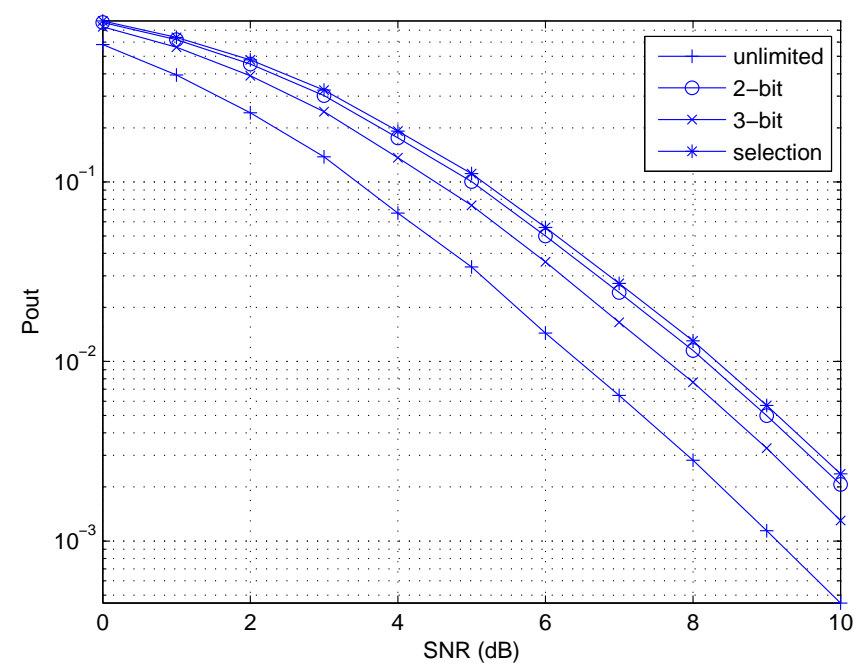

Fig. 2. Outage Probabilities for AF B-OC with $m=3, R=0.9$

codebooks achieve the same maximal received signal power, selection is the one that minimizes noise amplification.

Figure 2 shows the outage probability of these schemes in a network with 3 potential relays. All the channel gains in the network are i.i.d. $\mathcal{C N}(0,1)$ random variables. From the figures we can see the $1.95 \mathrm{~dB}$ performance loss between selection and B-UF as predicted in Table I. It also shows in the figure that compared to selection scheme, optimal beamforming with limited feedback achieves little improvement, while entailing significant system-wide disadvantages such as extremely high complexity and slow convergence rate for GLA and synchronization of relays. In other words, although increasing the amount of feedback and designing a nearoptimal codebook through the GLA can improve performance, this approach involves practical difficulties and yields only small performance gains.

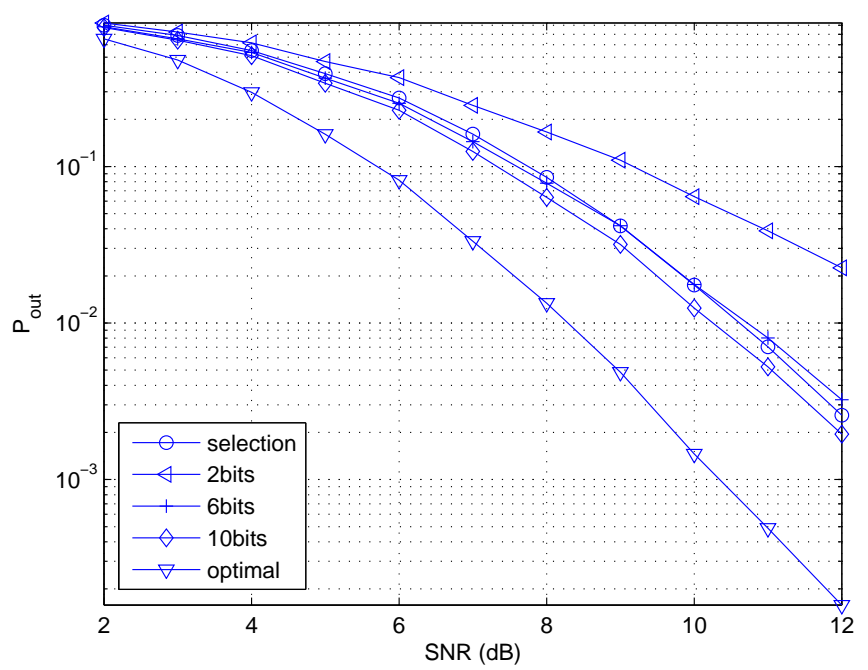

Fig. 3. Outage Probabilities for AF B-RF with $m=4, R=1.1$

\section{B. Random Beamforming with Limited Feedback}

The GLA described in the previous section has extremely high complexity (which grows dramatically with increasing $m$ ) and has to be run whenever the channel statistics changes. It is thus impractical for implementation. If we assume i.i.d. channels among the network, a random codebook generated from the uniform distribution on the complex unit sphere can be used instead. It has been proven that in MIMO systems with such a random codebook, the gap to capacity [10] and outage probability [8] between beamforming with limited and unlimited feedback can be reduced by increasing the number of feedback bits. In this section we study the random codebook approach in AF networks by first showing the simulation results.

Figure 3 shows the outage performances of an AF network with 4 relay nodes. It shows that, as expected, B-RC performs worse than the selection AF scheme with the same amount of feedback. In fact, the AF B-RC scheme requires about 4 bits more feedback to achieve the same performance at $P_{\text {out }}=$ $10^{-2}$ as the selection scheme. Figure 4 shows that when the number of relay nodes increases to 8 , the extra feedback for B-RC increases to about 6 bits.

From Section IV-A we know that, due to the noise amplification, AF beamforming imposes different requirements on codebook design than transmit beamforming in a traditional MISO system. In a MISO system where the channel is random to the transmitter, there is no bias in any "direction" and the optimal codebook design is to choose vectors on the unit sphere with maximum separation in terms of Euclidean distance. This translates to using the angle between vectors as the design parameter to be maximized. With AF networks, on the other hand, the noise amplification distorts the unit sphere and, so, rotations of codebooks are not equivalent. In particular, as shown in (10), since the optimal beamforming vector is distorted by the factor $\left\|\left(\mathbf{I}+\mathbf{H H}^{\dagger}\right)^{-1}\right\|$ instead of just matched filtering with the channel vector $\mathbf{h}$, the angle between code vectors is no longer the optimal metric for 


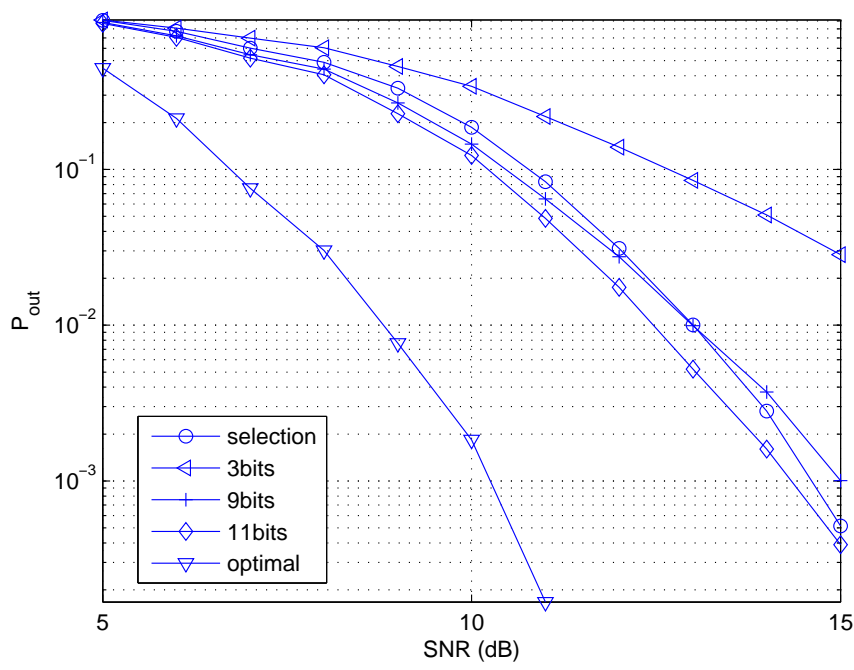

Fig. 4. Outage Probabilities for AF B-RF with $m=8, R=1.7$

performance. Note however that this distortion of the unit sphere is a function of the channel realization, in turn making the average performance hard to analyze.

Figures 3 and 4 also suggest a loss in diversity order for the B-RC scheme. Although it is difficult to prove the loss of diversity analytically, a plausible cause can be postulated: Due to the noise amplification effect of the AF relays, even in the high SNR regime, there is still a non-zero probability for the equivalent received noise power to be large. The outage events occur either when the channel condition is bad or the equivalent noise power is large, thus the diversity order of the outage probability decreases. Note that, from (15) selection achieves the maximum diversity order of $m+1$.

Since the selection scheme outperforms the AF B-RC by requiring less feedback, without diversity loss and operates asynchronously, we can claim that the selection scheme is the sub-optimal practical scheme of choice for AF networks with limited feedback. In other words, for AF networks with limited feedback, we would suggest the selection scheme, if the amount of available feedback is about $\log _{2}(m)$, for its excellent performance and the significant advantage of not requiring synchronization across relays.

\section{CONCLUSION}

The relay selection scheme has been proven to outperform the repetition-based relaying scheme in multiple relay $\mathrm{AF}$ networks. In this paper, we treat relay selection scheme as a special case of relay beamforming with limited feedback of $\log _{2} m$ bits and beamforming vectors as columns of the identity matrix. Transmit beamforming with limited and unlimited feedback has been studied in MISO systems, where matched filtering is the optimal beamforming with unlimited feedback, and the Grassmanian Line Packing gives the optimal codebook design for limited feedback. In AF networks, however, these two schemes are no longer optimal due to noise amplification at the relay nodes. We find the performance loss of selection compared to optimal relay beamforming with unlimited feedback by deriving the outage probability and error probability of both schemes.

In the case of limited feedback, although beamforming with optimal codebook has the best performance, the codebook design is very complex and therefore impractical. By comparing selection with another practical scheme, beamforming with random codebooks, we realize that selection has stronger performance and the advantage of not requiring synchronization. Therefore the selection scheme is probably the most attractive scheme for AF networks with limited feedback currently known.

\section{REFERENCES}

[1] J. N. Laneman and G. W. Wornell, "Distributed space-time-coded protocols for exploiting cooperative diversity in wireless networks," IEEE Transactions on Information Theory, vol. 49, pp. 2415-2425, November 2003.

[2] E. Beres and R. Adve, "On selection cooperation in distributed networks," Proceedings of IEEE 40th Conference on Information Science and Systems, March 2006.

[3] Y. Zhao, R. Adve, and T. J. Lim, "Improving amplify-and-forward relay networks: Optimal power allocation versus selection," Proc. IEEE International Symp. on Information Theory, July 2006.

[4] Y. Zhao, R. Adve, and T. J. Lim, "Symbol error rate of selection amplifyand-forward relay systems," IEEE Communications Letters, vol. 10, pp. 757-759, November 2006.

[5] A. Bletsas, A. Khisti, D. P. Reed, and A. Lippman, "A simple cooperative diversity method based on network path selection," IEEE Journal on Selected Areas in Communicaitons, vol. 24, pp. 659-672, March 2006.

[6] Z. Yi and I.-M. Kim, "Joint optimization of precoders and decoders with partial channel side information in cooperative networks," accepted to IEEE Journal on Selected Areas of Communications, 2006.

[7] D. J. Love, R. W. Heath, and T. Strohmer, "Grassmannian beamforming for Multiple-Input Multiple-Output wireless systems," IEEE Transactions on Information Theory, vol. 49, pp. 2735-2747, October 2003.

[8] K. K. Mukkavilli, A. Sabharwal, E. Erkip, and B. Aazhang, "On beamforming with finite rate feedback in multiple-antenna systems," IEEE Transactions on Information Theory, vol. 49, pp. 2562-2579, October 2003.

[9] W. Santipach and M. L. Honig, "Achievable rate for MIMO fading channels with limited feedback and linear receivers," Proc. IEEE Int. Symp. on Spread-Spectrum Tech. Appl. (ISSSTA), 2004.

[10] A. D. Dabbagh and D. J. Love, "Feedback rate-capacity loss tradeoff for limited feedback MIMO systems," IEEE Transactions on Information Theory, vol. 52, pp. 2190-2202, May 2006.

[11] G. H. Golub and C. F. Van Loan, Matrix Computations: Third Edition. The John Hopkins University Press, 1996.

[12] S. Zhou, Z. Wang, and G. B. Giannakis, "Quantifying the power loss when transmit beamforming relies on finite-rate feedback," IEEE Transactions on Wireless Communications, vol. 4, pp. 1948-1957, July 2005.

[13] A. Gersho and R. M. Gray, Vector Quantization and Signal Compression. Kluwer Academic Publishers, 1992. 